\title{
A TOMOGRAPHIC OCEAN SOUND SPEED PROFILE N53 FROM A LONG VERTICAL ACOUSTIC ARRAY
}

by

\author{
JAMES MURWANTHANJE NJERU \\ B.S.E.E., Lafayette College (1989) \\ B.S.(Physics), Lafayette College (1989) \\ SUBMITTED IN PARTIAL FULFILLMENT \\ OF THE REQUIREMENTS FOR THE DEGREE OF \\ MASTER OF SCIENCE \\ at the \\ MASSACHUSETTS INSTITUTE OF TECHNOLOGY \\ and \\ WOODS HOLE OCEANOGRAPHIC INSTITUTION
}

February 1992

(C) James Murwanthanje Njeru, MCMXCII. All rights reserved.

The author hereby grants to MIT and to WHOI permission to reproduce and to distribute copies of this thesis document in whole or in part.

Author

Department of Electrical Engineering, Massachussetts Institute of Technology, and the Joint Program in Oceanography and Oceanographic Engineering,

Massachusetts Institute of Technology/Woods Hole Oceanographic Institution

November 27, 1991

Certified by

John L. Spiesberger

Associate Skientist, WHOI, Thesis Supervisor

Certified by

Alan V. Oppenheim

Distinguished Professgr of Electrical Engineering, MIT, Thesis Supervisor

Accepted by

Arthur Bangoggeroer

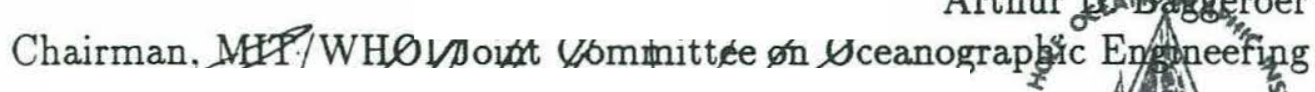

Accepted by

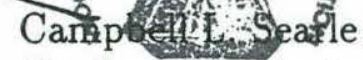

Chăr, MIT EEC S'Department Committee on Graduate'students 


\title{
A Tomographic Ocean Sound Speed Profile from a Long
}

Vertical Acoustic Array

by

\author{
James Murwanthanje Njeru
}

\author{
Submitted in partial fulfillment of the \\ requirements for the degree of \\ Master of Science \\ at the \\ MASSACHUSETTS INSTITUTE OF TECHNOLOGY \\ and the \\ WOODS HOLE OCEANOGRAPHIC INSTITUTION
}

February 1992

\begin{abstract}
An average sound speed profile over a $1000 \mathrm{~km}$ section of the northeast Pacific ocean is obtained using Ocean Acoustic Tomography, from data acquired during the 1987 SVLA experiment on a long $(900 \mathrm{~m}) 120$ hydrophone vertical acoustic array.

In particular, we pulse compress the received signal with a phase-only matched filter. The signal, centered at $80 \mathrm{~Hz}$, is phase-modulated by a maximal length sequence. A fast m-sequence cross-correlation algorithm based on the Hadamard transform is used. In addition, wide band Doppler correction and coherent averaging of repetitions of the signal are performed.

The tomographic inversion is initialized from a range averaged climatological profile. Multipaths are identified from ray theory. The identified arrivals are inverted for a range-independent sound speed profile change estimate. Estimates of source and array position error are also obtained. For the limited data set used, the sound speed change estimate is found to be insignificant, and a significant instrument position estimate is obtained.
\end{abstract}

Thesis Supervisor: John L. Spiesberger

Title: Associate Scientist, WHOI

Thesis Supervisor: Alan V. Oppenheim

Title: Distinguished Professor of Electrical Engineering, MIT 


\section{Acknowledgments}

My biggest thanks goes to John Spiesberger for his encouragement, support and patience. Without his deep insight and resourcefulness, this thesis would never have been completed. I am also greatly indebted to Dr. Bruce Cornuelle of Scripps Institution of Oceanography who provided the inverse routines and with whom I spent many hours on the phone porting them onto the Suns. I thank SAIC, Inc., McLean VA, for the data from the $S V L A$ experiment; specifically, Dr. Peter Mikhalevsky, Herb Freese and Dave Gever who were extremely helpful. I also thank Dr. Kurt Metzger of the Communications and Signal Processing Lab, University of Michigan, for providing reception processing routines.

There are many people from whom I learned a lot. I would first like to express my deepest appreciation to Jim Bowlin at WHOI, who endured my questions throughout. I thoroughly enjoyed working with Tim Duda, who kindly allowed me the use of his Sun, fellow graduate students Rich Pawlowicz and John Nystrom, and many others at WHOI.

At MIT, Al Oppenheim provided invaluable advice and a most stimulating environment to work in; namely, the Digital Signal Processing Group. It is impossible to express the indelible impression that DSPG members John Buck, Steve Isabelle, Andy Singer, Jim Preisig, Greg Wornell, Paul Beckman, Steve Scherock, Saurav Bhatta and others, made on me during the last two years. You guys were excellent to work with. 


\section{Contents}

Introduction $\quad 4$

1 Description of experiment 6

1.1 Experiment geometry and navigation $\ldots \ldots \ldots \ldots$

1.2 The transmitted signal . . . . . . . . . . . . . . . . . 10

2 Signal processing $\quad 11$

2.1 Description . . . . . . . . . . . . . . . . 11

2.2 Maximal length sequences . . . . . . . . . . . . . . 12

3 Ray tracing and multipath identification $\quad 15$

3.1 Ray Tracing . . . . . . . . . . . . . . . . . . 15

4 Tomographic inversion $\quad 19$

4.1 Forward problem linearization . . . . . . . . . . . . . . . 19

4.2 Inversion . . . . . . . . . . . . . . . . . . . 24

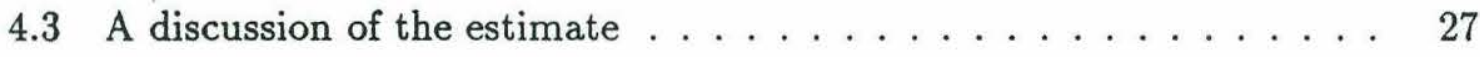

A The Gauss Markov theorem $\quad 29$

B 3-D parameterization $\quad 32$ 


\section{List of Figures}

1-1 Geographical region of experiment . . . . . . . . . . 7

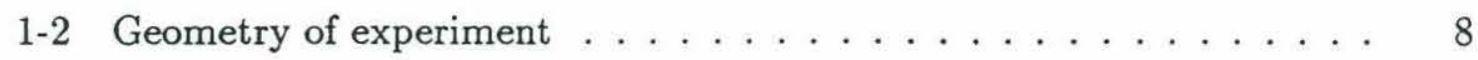

1-3 Range averaged sound-speed profile . . . . . . . . . . . 9

2-1 Processed reception and predicted arrival time structure $\ldots \ldots \ldots$

2-2 Maximal length sequence generator . . . . . . . . . . . . . 14

3-1 Range independent ray trace . . . . . . . . . . . . 17

4-1 Sound speed change estimate and standard deviations . . . . . . . . 27 


\section{List of Tables}

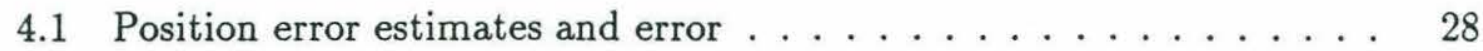




\section{Introduction}

Ocean acoustic tomography is a technique where travel time measurements in the ocean are used to infer ocean properties such as temperature and current velocity. Large-scale fluctuations of temperature in the ocean affect the weather, climate, ocean circulation and the distribution of marine organisms [1]. Ocean temperature over horizontal distances has traditionally been obtained from point measurements with instruments such as CTDs (conductivity-temperature-depth) and XBTs (expendable bathythermograph). However, point measurements can only sample the ocean at a finite number of locations, the limitation being the cost and effort required to deploy these instruments. Since point measurements of the horizontal variation in sound speed sample the ocean sparsely, they yield aliased versions of the true horizontal spectrum. Additionally, point measurements are difficult to obtain simultaneously in time. For example, a CTD survey involves a ship traversing the path of interest, deploying CTD instruments at points along the path. But, since sound travels at about $1500(\mathrm{~m} / \mathrm{s})$ in the ocean, tomographic measurements of travel times, are virtually instantaneous. And, because they are integral, tomographic measurements of travel times act as a low-pass filter, attenuating the contaminating high frequency spectral components present in point measurements [1], [2].

In the 1987 SVLA (Short Vertical Line Array) experiment, multiple transmissions were heard at a 120 hydrophone, $900 \mathrm{~m}$ long vertical acoustic array. It is the first experiment in which acoustic time fronts have been observed at long range [3]. In the context of tomography, the expectation that the number and diversity of multipaths would yield high spatial resolution motivates this work. A preliminary thermal map of a $1000 \mathrm{~km}$ section of the northeast Pacific ocean is obtained using acoustic tomography, from a small part of the data acquired during the 1987 SVLA experiment. Details of the experiment geometry, navigation, and signal characteristics, are discussed in chapter 1.

The transmitted signal is centered at $80 \mathrm{~Hz}$ and phase-modulated by a maximal length sequence. The reception is processed to pulse compress the received signal 
using a phase-only matched filter. A discussion of the signal decoding, particularly Metzger's Moving Ship Tomography (MST) code, and a brief summary of the properties of $\mathrm{m}$-sequences and their generation are in chapter 2.

In tomography, multipaths are usually identified from ray theory. In chapter 3, we briefly discuss ray theory, the program we use to compute the ray paths and travel times, and illustrate the identification.

In chapter 4 , we discuss the inversion of the identified arrivals for a range-independent sound speed estimate, using Cornuelle's three-dimensional inverse routines. Estimates of source and receiver position are also obtained. We present and discuss the estimates at the end of the chapter. 


\section{Chapter 1}

\section{Description of experiment}

\subsection{Experiment geometry and navigation}

A vertical $900 \mathrm{~m}$ long acoustic array was deployed from the research platform FLIP in the northeast Pacific during September, 1987. The geographical region in which the experiment was performed is shown in figure 1-1 [3]. The 120 array elements were spaced at $7.5 \mathrm{~m}$, with the top element at $400 \mathrm{~m}$ depth (figure 1-2). The source, at $1000 \mathrm{~km}$ range, was suspended at $150 \mathrm{~m}$ in the deep ocean $(5000 \mathrm{~m})$.

Source and receiving array navigation was done using near-bottom acoustic transponders. The reference position of the source was determined from the GPS position of the Research Vessel Desteiguer, from which the source was suspended. Here, only GPS data are used to determine source position. The accuracy of the source position $(x-y)$ is $50 \mathrm{~m}$ [3]. We use the nominal $150 \mathrm{~m}$ source depth which is accurate to a few meters.

The reference position of FLIP was determined from the GPS position of a ship close by. The navigation data available gave the position of FLIP to an accuracy of $1500 \mathrm{~m}$ and the array never drifted more than $30 \mathrm{~m}$ away from FLIP. The array position ( $\mathrm{x}-\mathrm{y})$ is known to $2.5 \mathrm{~m}(\mathrm{rms})$ relative to FLIP. We use $1500 \mathrm{~m}$ as the overall uncertainty in array position. Because the array is known not to tilt beyond $2^{\circ}$, we assume that it remains vertical. The array element depth is known to $1.5 \mathrm{~m}$ (rms). For this preliminary work, we used only receptions at the top $(439 \mathrm{~m})$, middle ( 888 


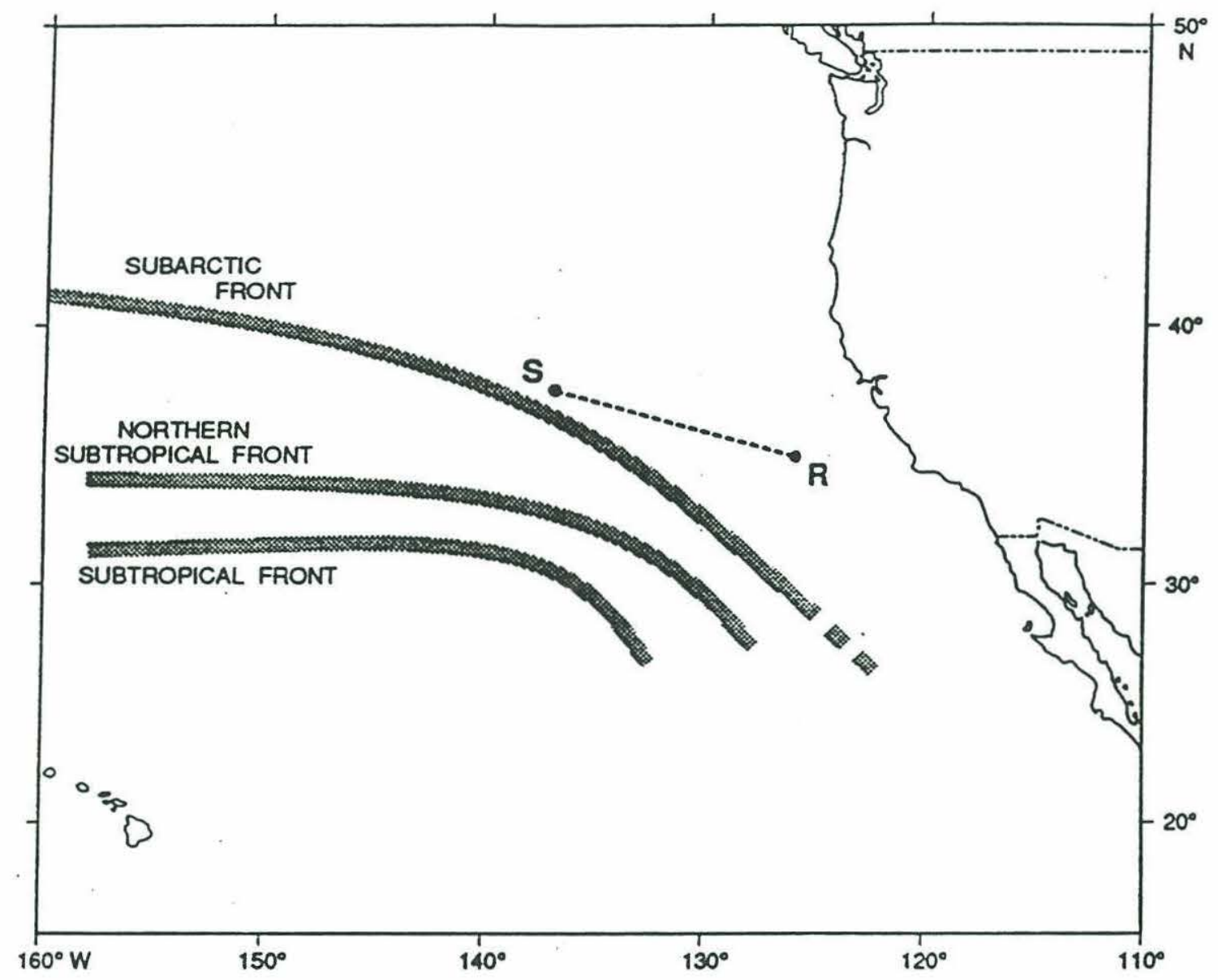

Figure 1-1: Geographical location of the SVLA experiment. The shaded areas indicate the range variability of the fronts. The transmission is not expected to pass through any frontal region. Taken from Sparrock [3]. 


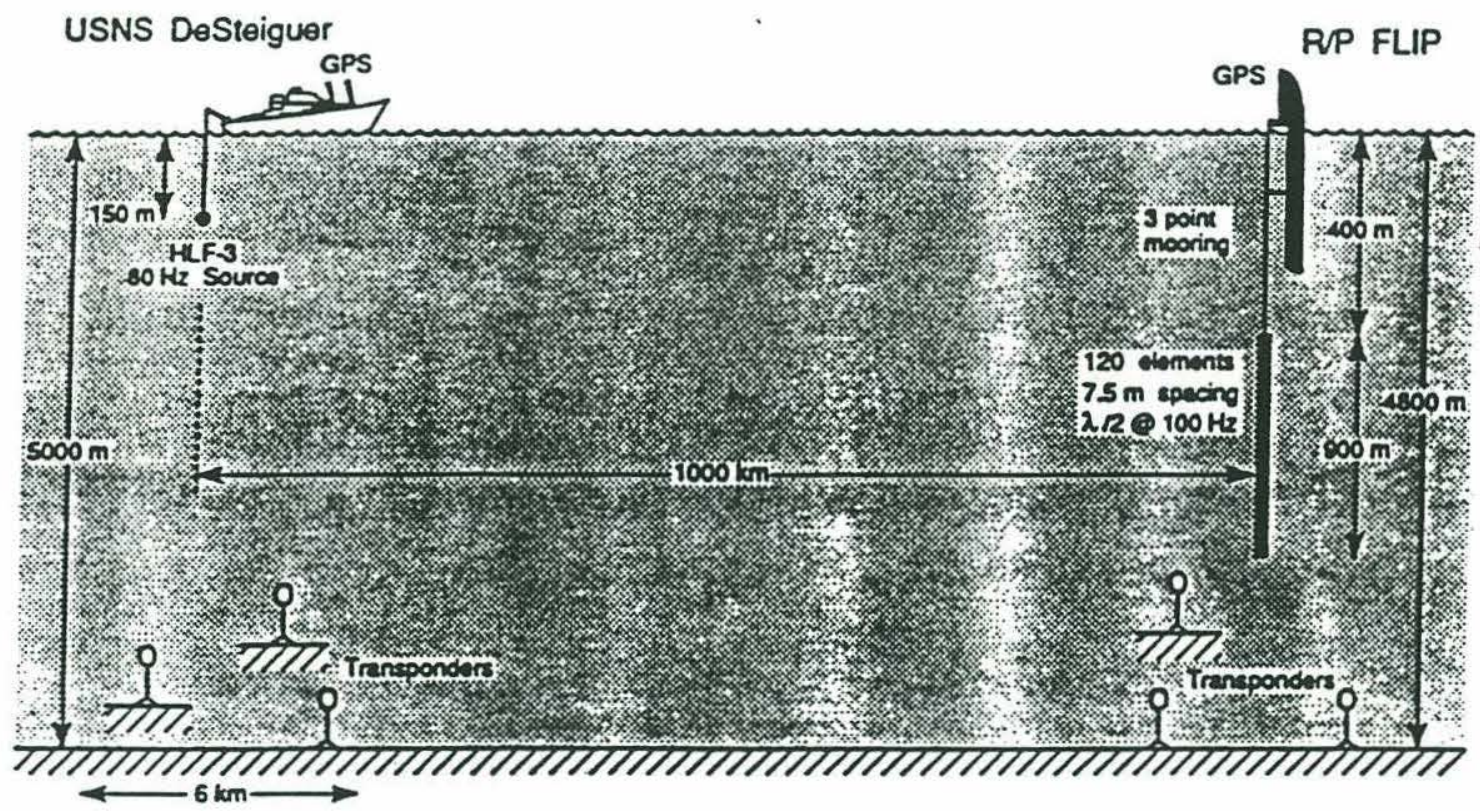

Figure 1-2: Geometry of experiment. Taken from Sparrock [3]. 

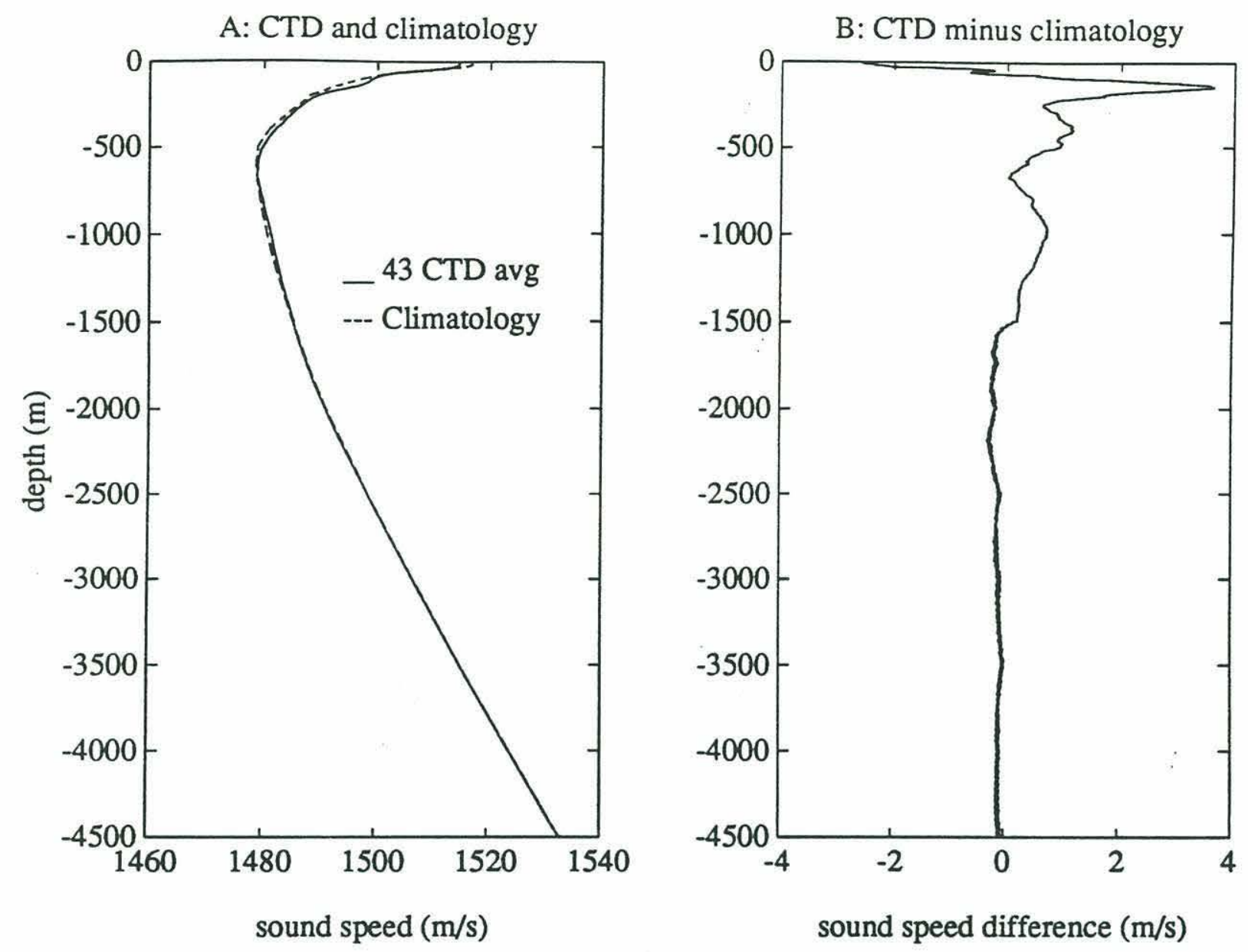

Figure 1-3: A: Range averaged sound speed profile derived from CTD casts during the experiment. The Chen-Millero algorithm [24] is used to convert the CTD data to sound speed. The dashed line is the Levitus [22] database summer climatology converted to sound speed using the Del Grosso algorithm [23].

$B$ : The difference between the two profiles in $A$.

$\mathrm{m})$, and bottom $(1331 \mathrm{~m})$ hydrophones in the array. Further detail on the array geometry and navigation can be found elsewhere [3], [4], [5].

Various environmental data were collected during the experiment, the most accurate being the conductivity-temperature-depth (CTD) profiles [3]. The CTD profiles were initially sampled densely. They were smoothed using a half meter half-width cosine squared window and resampled at $1 \mathrm{~m}$ spacing [3]. The Chen-Millero algorithm [24] was used to convert the CTD measurements to sound speed profiles. Figure 1-3 shows a plot of the range-averaged sound speed profile obtained from CTD measurements along the experimental section. The climatological profile, used as the reference 
for the inverse, and discussed later in this work, is also shown.

\subsection{The transmitted signal:}

Nine periods of a $20 \mathrm{~Hz}$ bandwidth ( $3 \mathrm{~dB}$ point), phase-modulated, 255 digit $\mathrm{m}$-sequence signal were transmitted at hourly intervals. The center frequency was $80 \mathrm{~Hz}$ and there were 4 carrier cycles per digit. The carrier modulation angle used $\left(86.41688^{\circ}\right)$ maximized the signal to noise ratio of the processed reception [7]. Explicitly, the transmitted signal has the form

$$
s(t)=\cos \left(2 \pi f_{c} t+b_{i} \theta\right)
$$

where $f_{c}$ is the center frequency, $t$ is time, $\theta$ is the modulation angle and $b_{i}$ are the $( \pm 1) \mathrm{m}$-sequence values. The $b_{i}$ are held constant for 4 carrier cycles, the process continuing until the set of $b_{i}$ have been repeated 9 times. The time resolution is 50 ms. Each period is $12.75 \mathrm{~s}$ long and the entire signal is $114.75 \mathrm{~s}$ long. The sequence law was 537 (octal) and the sequence generator was initialized at (10000000). 


\section{Chapter 2}

\section{Signal processing}

\subsection{Description}

The acoustic transducers used in the ocean are limited in the amount of energy that they can emit in a single pulse [7]. Sending longer duration linear maximal sequence (m-sequence) modulated signals, rather than a single pulse of the same duration as one digit of the m-sequence, increases the signal to noise ratio (SNR) at the output of the matched filter used to process the reception; specifically, the SNR increases by a factor of $L$, the number of digits in the m-sequence [7]. M-sequences are two state sequences that possess randomness properties similar to white noise and, in particular, have a two valued autocorrelation function [7], [10]. The crosscorrelation involves evaluating only additions and subtractions (ie., no multiplications) as the sequences have binary $( \pm 1)$ values. M-sequences are easy to generate and the next section summarizes the generation of the m-sequence used in this experiment. More significant, however, is the existence of a fast algorithm to perform the cross-correlations [11], [12]. Computing the cross-correlation between $n$ real values and an m-sequence of length $n$ directly, in the time domain, requires $n(n-1)$ operations (additions and subtractions) while the fast algorithm based on the fast Hadamard transform reduces this to $n \log _{2} n$ operations [12].

Metzger's Moving Ship Tomography program (MST) is used to process the reception. First, 8 of the 9 repetitions of the signal are averaged. Averaging 8 rather than 
9 repetitions avoids end effects. This improves the SNR by a factor of 8 , the number of repetitions averaged [7]. The signal is then demodulated by down shifting the frequency content to baseband and lowpass filtering. An interpolation is then done to ensure that there are an integer number of demodulates per digit. This makes it possible to do the rest of the processing in parallel (ie. using a one sample per digit description for each stream). The data is divided into separate streams. The first stream contains the first demodulate in each digit, the second contains the second demodulate in each digit and so on. Each stream is treated identically in what follows.

The crosscorrelation consists of a prescrambling of the input stream, performing the Hadamard transform using the fast Hadamard transform algorithm and then postscrambling the result. The streams are then put back in their original order. Since all the operations are additions and subtractions, the real and imaginary parts are processed separately. That is, for each stream, a correlation is done for the real part and another for the imaginary part and the results combined.

If Doppler correction is to be done, a time expansion or compression is performed after the demodulation, followed by a linear interpolation. Doing the Doppler expansion at this point reduces error as the demodulated signal is varying much less rapidly. It is also necessary to account for the fact that the demodulation frequency used was incorrect. A time expansion rather than a simple frequency shift is needed because our signal is broadband. MST will search over a specified range of velocities to find the Doppler shift that yields the highest peak. Figure 2-1A shows the processed result for the reception at one hydrophone.

\subsection{Maximal length sequences}

Maximal length sequences (m-sequences), although deterministic, possess statistical properties similar to a series of Bernoulli trials with the two states being equally likely [10]. Figure 2-2 shows an 8-stage shift register configuration that can be used to generate the 255 digit m-sequence used in this experiment. The configuration has the form of the Modular Shift Register Generator (MSRG), a canonical form that is 

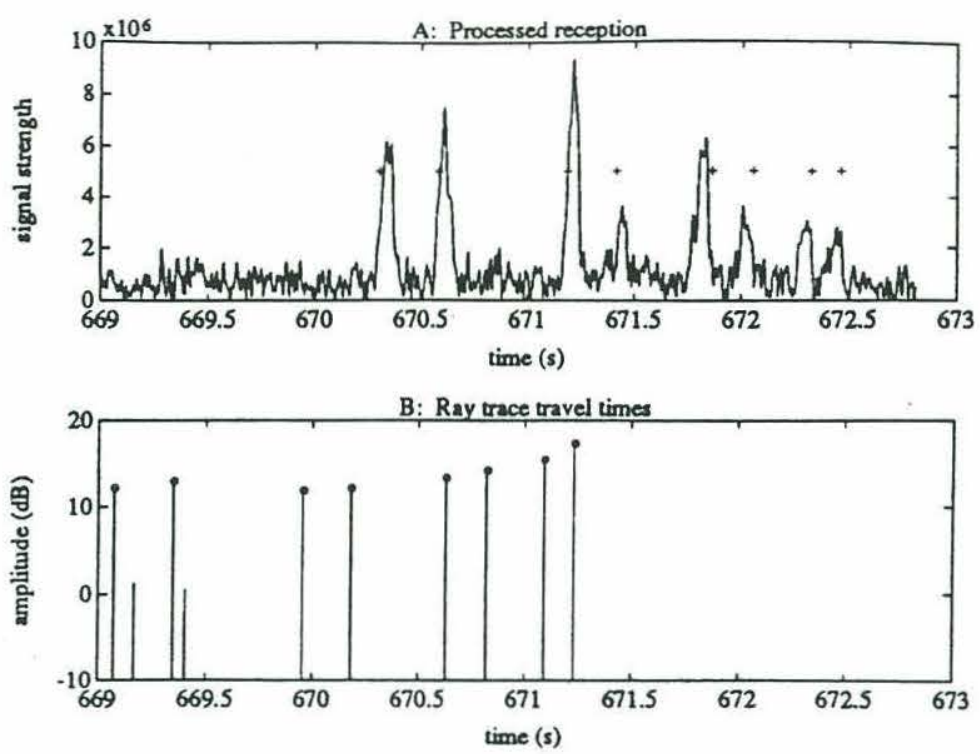

Figure 2-1: A: Processed reception for the middle hydrophone in the array. Eight periods of the transmission are processed to avoid end effects. A search over velocities yields zero velocity as the velocity giving the highest peak response.

$B$ : Arrival time structure for a range-independent ray trace between the source and the middle hydrophone in the array through the climatological sound speed profile of figure 1-3. The amplitudes of the ray trace differ from those of the reception. The + symbols in $A$ show the positions of the arrivals in $B$ with all the arrivals right shifted by the same amount and arrivals that do not fall on a peak removed.

easy to program [13].

The sequence law (537, octal), indicates which of the switches $m_{0}$ through $m_{8}$ are closed; specifically, the law is rewritten as the binary number 101011111 (base 2), and the digits in this number labeled $m_{8}$ through $m_{0}$ respectively. That is, $m_{8}=1$, $m_{7}=0$ and so on. When an $m_{i}=1$ the switch is closed and when an $m_{i}=0$ it is left open, as indicated in figure 2-2. The blocks represent unit delay elements and the $\oplus$ represent mod 2 adders. Choices of switch settings that yield m-sequences for up to 19-stage shift register generators have been tabulated in [13]. References [10] and [9] discuss the theory behind m-sequences and their generation.

The contents of the registers may be initialized in any state (set of register values) except for the all zero state (in which the register would be trapped). In the SVLA experiment the initialization was 10000000 with the 1 in the leftmost register of figure 2-2.

The output can be taken from the output of any of the delay elements because 
each delay element contains delayed forms of the same m-sequence [10]. In figure 2-2, we take the output from the right-most delay element. These output values are then mapped so that 1 becomes -1 and 0 becomes +1 . This output sequence is labeled $b_{i}$.

The output sequence is periodic every $255=2^{8}-1$ digits. Because the generator goes through all possible states (except the all zero state) before repeating, the sequence is referred to as being of maximal length.

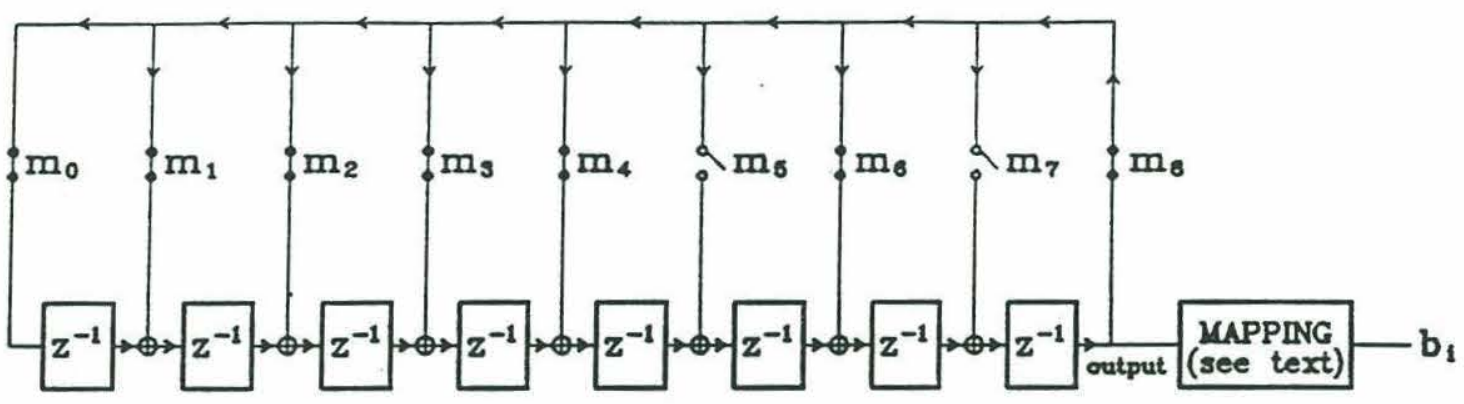

Figure 2-2: $\quad M S R G$ shift register configuration that could be used to generate the 537 sequence law m-sequence. The registers are initialized at, from right to left, 10000000. The mapping in the rightmost box is specified in the text.

Of particular relevance in the experiment is the two-level autocorrelation function of $\mathrm{m}$-sequences. This is a property associated with white noise. Defining the $\mathrm{m}$ sequence values as $b_{i}$, the sequence length as $P(=255$ here), the autocorrelation function can be shown to be [10],

$$
c(k) \equiv \frac{1}{P} \sum_{i=1}^{P} b_{i} b_{i+k}=\left\{\begin{array}{lll}
1 & k=0 & (\bmod P) \\
\frac{-1}{P} & 0<k<P & (\bmod P)
\end{array}\right.
$$

Notice that that the autocorrelation is not zero at $0<k<P$ as it would be for white noise. This offset is referred to as the dc error term and has to be corrected for [7]. 


\section{Chapter 3}

\section{Ray tracing and multipath identification}

\subsection{Ray Tracing}

Ocean sound speed increases with pressure, temperature and, to a much smaller extent, salinity (ocean currents also affect travel times to an even smaller extent). Sound speed profiles at mid-latitudes typically have high sound speeds near the surface, decreasing with depth because the influence of atmospheric heating decreases with depth. At greater depths the increasing pressure becomes dominant so that sound speed begins to increase with depth. The channel thus typically exhibits a sound speed minimum which, in our case, is at about $700 \mathrm{~m}$ (see figure 1-3). Close to the surface the profile is more structured due to processes such as surface-waveinduced mixing, mixing due to ocean currents and eddies.

Sound propagation through the ocean is described by the acoustic wave equation,

$$
\nabla^{2} p=\frac{1}{c^{2}} \frac{\partial^{2} p}{\partial^{2} t}
$$

where $p$ is the acoustic pressure, $c=c(x, z)$ is the sound speed, and $t$ is time. When the speed of sound does not vary rapidly over distances comparable to a wavelength [18] , that is, at high frequencies, the solution to the wave equation can often be 
described by ray theory. Rays can be shown to obey Snell's law,

$$
\frac{\cos \phi}{c(z)}=\text { constant }
$$

Here, $\phi$ is the angle the ray element makes with the horizontal and $c(z)$ is the vertically varying sound-speed. A useful result for the computation of ray paths is that within a linear $c(z)$ the ray is a circular arc [18] .

We expect that at $80 \mathrm{~Hz}$, acoustic travel times will be reasonably well modeled by ray theory [25].

Due to the distinct minimum in the sound channel, sound is trapped in the channel and ray paths form loops going from the source to the receiver. Because of the large distance $(1000 \mathrm{~km})$ between source and receiving array in this experiment, we do not expect bottom interacting rays to be heard at the array and we therefore do not trace them.

Rays leaving the source at a number of initial angles hit the receiver, with each of the rays having an associated travel time. Travel time is obtained by

$$
t_{i}=\int_{\Gamma(i)} \frac{d s}{c(s)}
$$

with $t_{i}$ being the travel time for the $i$ th ray, and $\Gamma(i)$ its path. $d s$ is an element of length along the path and $c(s)$ the sound-speed.

The identification process associates peaks in the processed reception with predicted arrival times and their corresponding ray paths computed by the ray tracing. This is illustrated in figures 2-1a,b. Fig 2-1b shows the arrival time structure predicted by the range-independent ray trace through the climatological profile of figure 1-3 between the source and the middle hydrophone in the receiving array. Figure 2-1a shows the processed reception at the same hydrophone. The processed reception is delayed about 1 second with respect to the predicted arrival time structure because the source-receiver separation used in the ray trace is inaccurate. A shifted version of predicted arrival time peaks is indicated by the + symbols in figure 2-1 a, with arrivals that do not correspond to peaks in the processed reception removed. Note that 


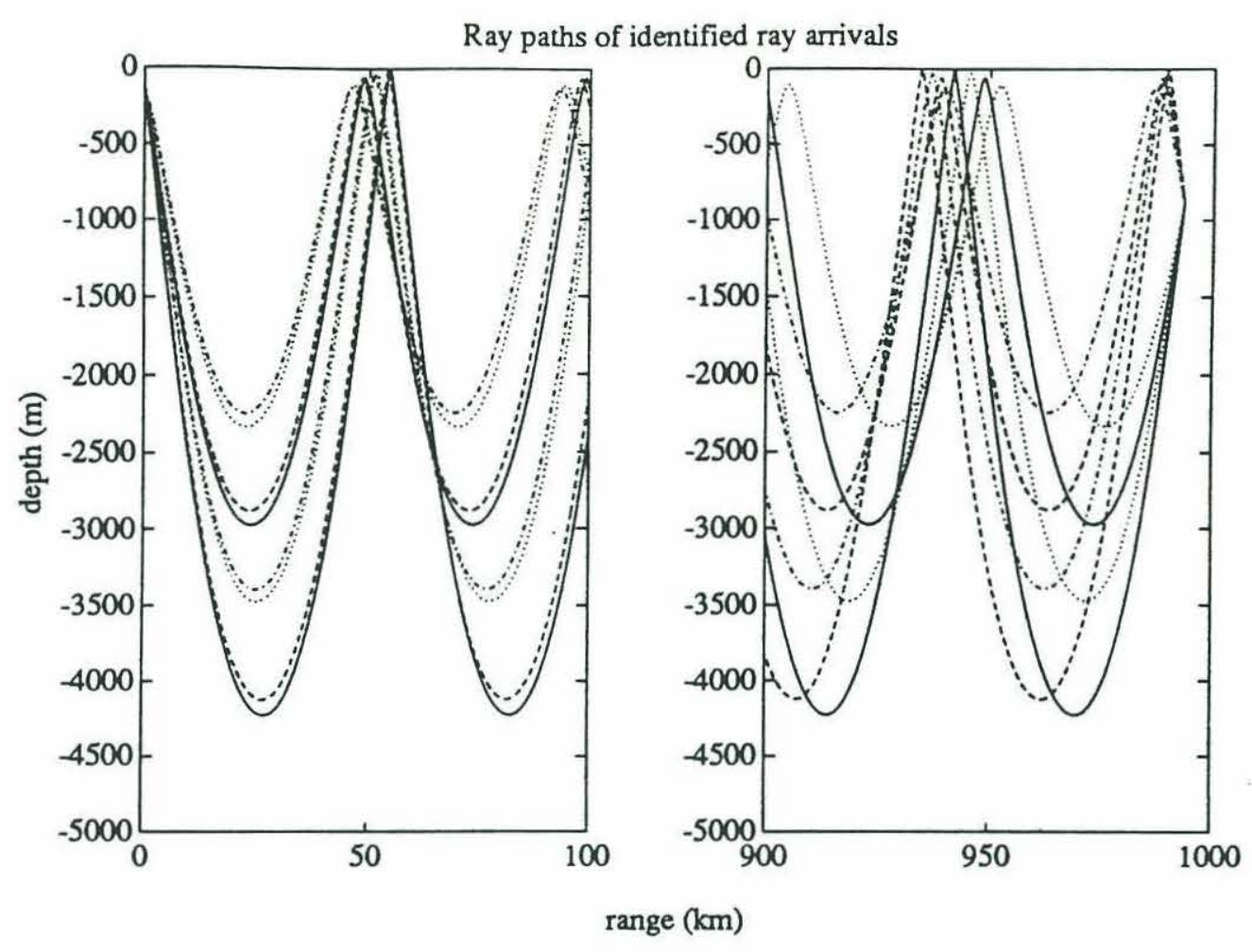

Figure 3-1: Range independent ray trace through a sound-speed profile derived from Levitus database climatology [22] using the Del-Grosso algorithm. Only the first and last $100 \mathrm{~km}$ are shown. Only rays identified in figure $2-1 \mathrm{~A}$ have been traced. It is coincidental that all the rays have negative initial angle.

the amplitudes predicted by the ray trace differ from the amplitudes of the reception.

Figure 3-1 shows the first and last $100 \mathrm{~km}$ of the ray paths corresponding to the identified arrivals in fig 2-1a. It is coincidental that all the rays in the figure have negative initial angles. Each ray path samples the ocean to a different depth, providing vertical resolution in the tomographic map.

In their seminal paper, Munk and Wunsch [2] showed that ocean acoustic tomography is a nonlinear problem because ray paths depend on the sound speed field [1]. However, the tomographic inversion may be initialized from an estimate of rays traced through the time independent sound speed component of the sound speed field $[1,14,15,16]$.

In this thesis, the tomographic inversion is initialized by tracing rays through a range-independent sound speed profile. In obtaining the profile (figure 1-3), the Del Grosso algorithm [23] is used to generate the sound speed profile from the climato- 
logical estimates of summer temperature and salinity along the transmission path. Reference [6] shows that the Del Grosso algorithm predicts sound speeds that are in agreement with those derived through the Chen-Millero algorithm [24] near the ocean surface, but predicts sound speeds that differ significantly from those derived through the Chen-Millero algorithm at greater ocean depths. We compare a sound speed map initialized from the reference profile to the range averaged sound speed profile derived from CTD data of figure 1-3.

We generate ray traces through range-independent sound speed profiles using a computer code due to Bruce D. Cornuelle (private communication). The World Geodetic System 1984 (WGS84) reference ellipsoid [6] is used to compute the sourcereceiver distance. The ray traces are done in a Cartesian coordinate system obtained by performing the Earth flattening transformation [6].

The sound speed profile is approximated as constant gradient horizontal layers, leading to ray paths that are circular arcs within the layers. The program computes geometric rays, and their travel times and amplitudes. Diffracted arrivals are not computed. 


\section{Chapter 4}

\section{Tomographic inversion}

\subsection{Forward problem linearization}

In the geographical region in which our experiment was performed, sound speed changes produce much stronger changes in travel time than ocean currents do. Therefore, since only sound speed maps are of interest, modeling ocean currents as part of the noise in the forward model does not introduce significant error. Ocean currents are modeled as part of the noise here.

As stated previously, travel time perturbations are, in general, nonlinear in sound speed perturbation. As is standard practice, to linearize the problem, the overall sound speed, $C$, is written as the sum of a reference state, $C_{o}$, and a perturbation, $\delta C$,

$$
C(x)=C_{o}(x)+\delta C(x)
$$

where $\left(|\delta C| \ll\left|C_{o}\right|\right)$. The subscript $o$ indicates the reference state and $x$ the range measured from source to receiver. A Taylor expansion of $\frac{1}{C}=\frac{1}{C_{o}+\delta C}$ yields the approximate travel time through the perturbed ocean,

$$
T_{k} \approx \int_{\Gamma(k)} \frac{d s}{C_{o}(s)}-\int_{\Gamma(k)} \frac{\delta C(s) d s}{C_{o}^{2}(s)}
$$


where $s$ is the distance along the ray path. The first term on the right is the travel time through the unperturbed ocean and the second is change in travel time (perturbed minus unperturbed), $\delta T_{k}$, for ray $k$,

$$
\delta T_{k}=-\int_{\Gamma(k)} \frac{\delta C(s) d s}{C_{o}^{2}(s)}+\text { h.o.t }
$$

The higher order terms (h.o.t) are ignored in the approximation.

Equation 4.1 is linear in sound speed perturbation, $\delta C$. The forward problem is finding the $\delta T_{k}$ when $\delta C$ is known and deducing $\delta C$ from $\delta T_{k}$ is the inverse problem. It is the quantity $\left(\delta T_{k}-\right.$ h.o.t $)$ that is obtained from subtracting the predicted travel time from the experimentally measured travel time, so that the h.o.t measure the uncertainty in $\delta T_{k}$ due to the linearization.

The observed travel time contains errors due to acoustic noise. Reference [17] shows that the theoretical variance of the arrival time computed by a matched filter for a signal to noise ratio $S / N^{1}$ and bandwidth $\Delta f(\mathrm{~Hz})^{2}$ is given by,

$$
\sigma_{\delta T}^{2}=\frac{1}{(S / N)(\Delta f)^{2}}
$$

For our experiment, $\sigma_{\delta T} \approx 4(m s)$. Clock errors $(\approx 1(m s))$ also contribute to the uncertainty in the observed travel time.

The accuracy of ray tracing is limited by the fact that ray tracing is an infinite frequency approximation and, when its done in 2-D, further by the fact that horizontal refraction is not accounted for [1]. For an $80 \mathrm{~Hz}$ signal and a range of $1000 \mathrm{~km}$, the uncertainty in the reference travel time due the infinite frequency approximation is about $60 \mathrm{~ms}$ [25]. In the region of the SVLA experiment, the uncertainty in the reference travel time due to horizontal refraction, for a range of $1000 \mathrm{~km}$, is about 2 $\mathrm{ms}[1]$.

\footnotetext{
${ }^{1} S / N$ is the ratio of the peak squared instantaneous output of the filter in the absence of noise, to the power of the filter output in the absence of the signal.

${ }^{2}(\Delta f)$ is the "spread" of the signal spectrum, specifically, $(\Delta f)^{2}=\frac{8 \pi^{2}}{E} \int_{0}^{\infty}\left(f-f_{0}\right)|S(f)|^{2} d f$, where $E$ is the energy in the real signal $s(t), f_{0}$ its center frequency, and $S(f)$ its spectrum.
} 
In addition, processes such as currents, eddy biases, internal wave biases, fluctuations of internal waves and tides are not incorporated into the computation of the reference travel time: rays are traced through an ocean devoid of these effects. Since these processes are not modeled in the inverse either, they are included as part of the uncertainty in reference travel time.

The uncertainty in reference travel time due to the limitations of 2-D ray tracing and ignorance of the contributions of the above processes, is estimated as in Spiesberger ([6], table II). Spiesberger's experiment, performed in the same geographical region as the $S V L A$ experiment, involved a source-receiver distance of $3000 \mathrm{~km}$, three times the source-receiver distance in the $S V L A$ experiment. That the two experiments involved different source-receiver distances is accounted for by appropriately scaling the reference travel time noise contributions of the 2-D ray tracing and the ocean processes above. A total reference travel time standard deviation of $67 \mathrm{~ms}$ is obtained for the SVLA experiment.

In Cornuelle's inverse routines, the sound speed perturbation is parameterized as an expansion in vertical and horizontal functions. Since the math is more cumbersome for the 3-D case, we discuss the 3-D parameterization in appendix B. The 2-D parameterization takes the form ${ }^{3}$,

$$
\delta c(x, z)=\sum_{i=1}^{L} a_{i 0} F_{i}(z)+\sum_{i=1}^{N} F_{i}(z)\left[\sum_{j=1}^{M} a_{i j} \cos \left(\frac{2 \pi j x}{R}\right)+b_{i j} \sin \left(\frac{2 \pi j x}{R}\right)\right]
$$

where $R$ is the periodicity of horizontal sinusoidal functions. Notice that $N$ vertical functions are allowed horizontal variation while $L-N$ are not $(L \geq N)$. For example, the errors due the sound speed algorithm would not be modeled as having horizontal variation.

Substituting this expansion in 4.1 and moving the integral inside the summations

\footnotetext{
${ }^{3}$ Strictly, the program allows $M$ to depend on the vertical function. In other words, $M$ could be replaced by $M_{i}$ in this equation, but this flexibility is left out here for simplicity.
} 
yields,

$$
\begin{aligned}
\delta T_{k}= & -\sum_{i=1}^{L} a_{i 0} \int_{\Gamma(k)} \frac{F_{i}(z(s)) d s}{C_{o}^{2}(s)}-\sum_{i=1}^{N} \sum_{j=1}^{M} a_{i j} \int_{\Gamma(k)} \frac{F_{i}(z(s)) \cos \left(\frac{2 \pi j x(s)}{R}\right) d s}{C_{o}^{2}(s)} \\
& -\sum_{i=1}^{N} \sum_{j=1}^{M} b_{i j} \int_{\Gamma(k)} \frac{F_{i}(z(s)) \sin \left(\frac{2 \pi j x(s)}{R}\right) d s}{C_{o}^{2}(s)}+n_{k}
\end{aligned}
$$

By dealing with each $j$ term individually one sees how this equation is rewritten in matrix form. Defining,

$$
\psi_{j}^{c}(k, i)=-\int_{\Gamma(k)} \frac{F_{i}(z(s)) \cos \left(\frac{2 \pi j x}{R}\right) d s}{C_{o}^{2}(s)}, \quad \psi_{j}^{s}(k, i)=-\int_{\Gamma(k)} \frac{F_{i}(z(s)) \sin \left(\frac{2 \pi j x}{R}\right) d s}{C_{o}^{2}(s)}
$$

and

$$
\boldsymbol{a}_{j}=\left[\begin{array}{c}
a_{1 j} \\
\vdots \\
a_{N j}
\end{array}\right], \quad \boldsymbol{b}_{j}=\left[\begin{array}{c}
b_{1 j} \\
\vdots \\
b_{N j}
\end{array}\right], \quad G_{j}^{c}=\left[\psi_{j}^{c}(k, i)\right], \quad G_{j}^{s}=\left[\psi_{j}^{s}(k, i)\right]
$$

then,

$$
d=\left[\begin{array}{lllllll}
G_{\circ}^{c} & G_{1}^{c} & \ldots & G_{M}^{c} & G_{1}^{s} & \ldots & G_{M}^{s}
\end{array}\right]\left[\begin{array}{c}
a_{\circ} \\
a_{1} \\
\vdots \\
a_{M} \\
b_{1} \\
\vdots \\
b_{M}
\end{array}\right]+n
$$

More succinctly, the forward problem becomes

$$
d=G_{a} m_{a}+n
$$

where, the travel time changes are contained in $d$, the model parameters, $a_{i j}$, in $m$, and the noise terms, in $\boldsymbol{n}$.

If the model parameters are uncorrelated with each other, the a priori model 
covariance matrix takes the diagonal $\mathrm{f}-\mathrm{nm}$,

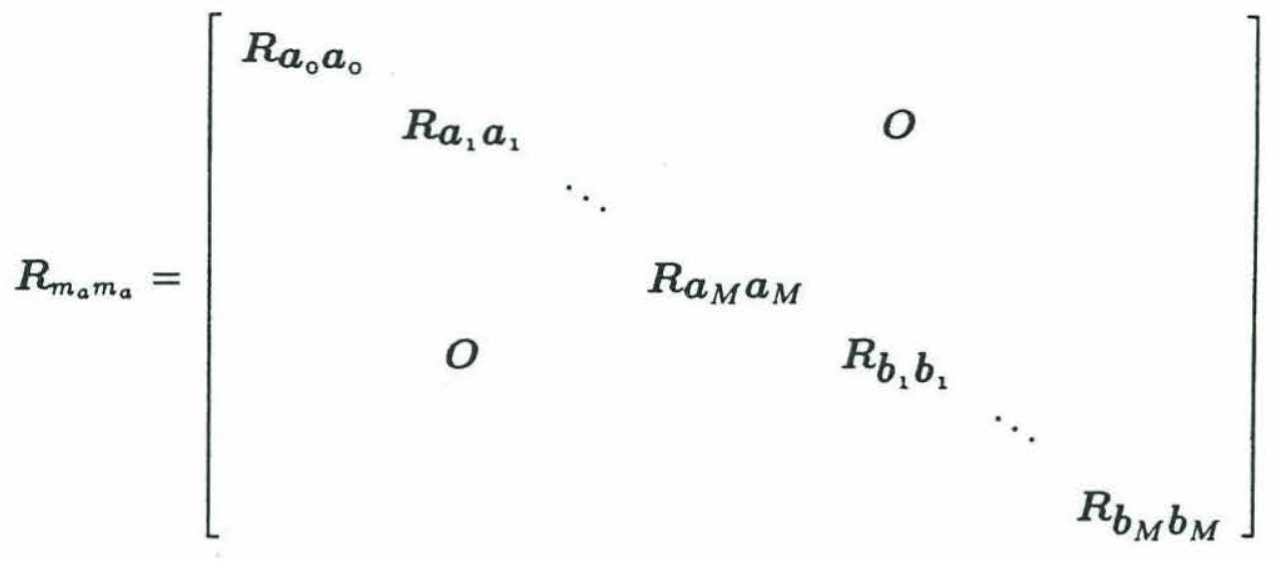

In this work, we use 40 vertical modes taken from Spiesberger [6], who also discusses the motivation for their use. We suppress the range dependence of the problem by specifying very small variances for the range dependent horizontal modes. That is, all the elements in $\boldsymbol{R}_{m_{a} m_{a}}$ except the elements in $\boldsymbol{R}_{a_{0} a_{o}}$ are set at very small values. 


\subsection{Inversion}

The travel time differences are inverted using Bruce Cornuelle's inversion routines which are capable of generating physical maps in three dimensions [26]. The inverse is based on the Gauss Markov theorem [20] (see appendix A) in which both the noise and model parameters are viewed as zero mean stochastic processes with known covariance matrices. Here, the model and noise are taken to be uncorrelated. A summary of the equations implemented in the inverse routines follows.

Let $G_{a}$ relate ocean model parameters $\boldsymbol{m}_{a}$ to travel time changes and let $G_{I}$ relate instrument position changes $m_{I}$ to travel time changes. The two contributions and a noise term $\boldsymbol{n}$ add up to the travel time change data $\boldsymbol{d}$,

$$
d=G_{a} m_{a}+G_{I} m_{I}+n
$$

or

$$
d=\left[\begin{array}{ll}
G_{a} & G_{I}
\end{array}\right]\left[\begin{array}{c}
m_{a} \\
m_{I}
\end{array}\right]+n
$$

which, concisely, is,

$$
d=G m+n
$$

The ocean parameters and the instrument position error parameters can therefore be estimated simultaneously by estimating $\boldsymbol{m}$. The Gauss Markov estimate (appendix A) of $\boldsymbol{m}$ is,

$$
\hat{\mathrm{m}}=R_{m m} G^{T}\left(G R_{m m} G^{T}+R_{n n}\right)^{-1} d
$$

However, Cornuelle's routines exploit the fact that the two components of $\boldsymbol{m}, \boldsymbol{m}_{\boldsymbol{a}}$ and $\boldsymbol{m}_{I}$, can be computed separately and conveniently. For example, the estimate of $m_{a}$ can be obtained by viewing equation 4.8 as

$$
d=G_{a} m_{a}+n_{1}
$$


where $\boldsymbol{n}_{1}$ is $\boldsymbol{G}_{I} \boldsymbol{m}_{I}+\boldsymbol{n}$.

$$
\boldsymbol{R}_{n_{1}}=E\left[\left(G_{I} m_{I}+n\right)\left(G_{I} m_{I}+n\right)^{T}\right]=G_{I} R_{m_{I} m_{I}} G_{I}^{T}+R_{n n}
$$

because $\boldsymbol{n}$ and $\boldsymbol{m}_{I}$ are uncorrelated. Again, the Gauss Markov solution for $\boldsymbol{m}_{a}$ is,

$$
\hat{\mathrm{m}}_{\mathrm{a}}=R_{m_{\mathrm{a}} m_{\mathrm{a}}} G_{a}^{T}\left(G_{a} R_{m_{\mathrm{a}} m_{\mathrm{a}}} G_{a}^{T}+G_{I} R_{m_{I} m_{I}} G_{a}^{T}+R_{n n}\right)^{-1} d
$$

or

$$
\hat{\mathrm{m}}_{\mathrm{a}}=R_{m_{\mathrm{a}} m_{a}} G_{a}^{T}\left(G R_{m m} G^{T}+R_{n n}\right)^{-1} d
$$

Similarly, viewing equation 4.8 as,

$$
d=G_{I} m_{I}+n_{2}
$$

where $\boldsymbol{n}_{2}$ is $\boldsymbol{G}_{a} \boldsymbol{m}_{a}+\boldsymbol{n}$, yields,

$$
\hat{\mathrm{m}}_{\mathrm{I}}=R_{m_{I} m_{I}} G_{I}^{T}\left(G R_{m m} G^{T}+R_{n n}\right)^{-1} d
$$

And, viewing equation 4.8 as,

$$
d=I n+n_{3}
$$

where $\boldsymbol{n}_{3}$ is $\boldsymbol{G}_{a} \boldsymbol{m}_{a}+\boldsymbol{G}_{I} \boldsymbol{m}_{I}$, and $I$ is the identity matrix, yields,

$$
\hat{\mathrm{n}}=\boldsymbol{R}_{n n}\left(G R_{m m} G^{T}+R_{n n}\right)^{-1} d
$$

Note that the estimate $\hat{\mathbf{n}}$ is the traditional residual, $\boldsymbol{d}-\boldsymbol{G} \hat{\mathrm{m}}$ (see appendix A).

Examining equations $4.11,4.15,4.17$ and 4.19 , one observes that they all contain the term $\left(G R_{m m} G^{T}+R_{n n}\right)^{-1}$. The term involves an inversion of a square matrix of size equal to the number of data points. But it need only be computed once and, by choosing the matrix to premultiply it by, one can selectively compute any of $\hat{\mathbf{m}}, \hat{\mathbf{m}} \mathbf{a}$, mi , or the residual $\hat{\mathbf{n}}$.

The covariance matrices were assumed diagonal. We have to be careful with 
$R_{m_{I} m_{I}}$ because the hydrophones are constrained to be part of an array and the hydrophone motion is therefore correlated. But, because we have only one receiver in our case, the instrument motion can be lumped into the source motion, by specifying a large position error standard deviation at the source and very small uncorrelated standard deviations at the hydrophones.

Using the process described to write down the estimates $\hat{\mathbf{m}}, \hat{\mathrm{m}}_{\mathbf{a}}$, and $\hat{\mathrm{m}}_{\mathbf{I}}$, the corresponding error covariances can be read off equation A.12 directly. For $\hat{m}$, the error covariance is

$$
C_{e e}=R_{m m}-R_{m m} G^{T}\left(G R_{m m} G^{T}+R_{n n}\right)^{-1} G R_{m m}^{T}
$$

For $\hat{m}_{\mathbf{a}}$, it is,

$$
C_{e e}=R_{m_{a} m_{a}}-R_{m_{a} m_{a}} G_{a}^{T}\left(G R_{m m} G^{T}+R_{n n}\right)^{-1} G_{a} R_{m_{a} m_{a}}^{T}
$$

and for $\hat{m}_{\mathbf{I}}$, it is,

$$
C_{e e}=R_{m_{I} m_{I}}-R_{m_{I} m_{I}} G_{I}^{T}\left(G R_{m m} G^{T}+R_{n n}\right)^{-1} G_{I} R_{m_{I} m_{I}}^{T}
$$

All these expressions all involve the term $\left(G R_{m m} G^{T}+R_{n n}\right)^{-1}$, which was common to the expressions for the estimates. 


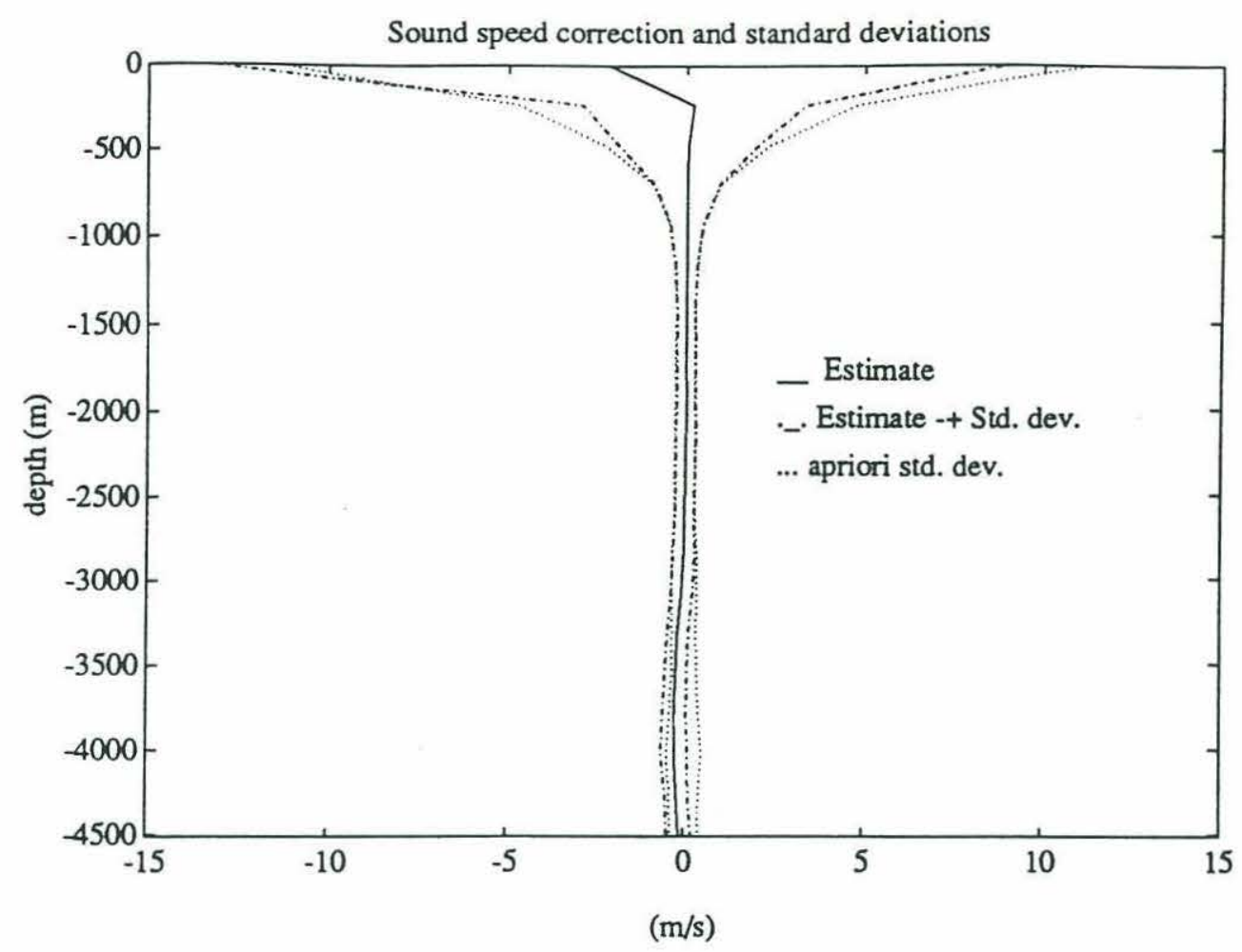

Figure 4-1: Sound speed change estimate, its standard deviation, and its a priori standard deviation. Arrivals at the top, middle, and bottom hydrophones for a single transmission were used in the inversion.

\subsection{A discussion of the estimate}

Figure 4-1 shows a plot of the sound speed profile change estimate, its standard deviation and the a prioristandard deviation. Arrivals at three hydrophones, sparsely spaced along the array, were used in the inversion. The climatological profile discussed previously was used to initialize the inversion.

The estimate is much smaller than the standard deviation and is thus not statistically significant. We have reduced the standard deviation of the estimate slightly, however, as it is smaller than the a priori standard deviation.

The instrument position correction estimates and standard deviations are shown in table 4.1. The a prioristandard deviations used are also shown. For each instrument, the coordinates are centered at the the original position. Because the hydrophones are constrained to be part of the array, we lump the position errors into the source location 


\begin{tabular}{||l|r|r|r||}
\hline \multicolumn{4}{||c||}{ a priori standard deviation (m) } \\
instrument (depth(m)) & $x$ & $y$ & $z$ \\
\hline \hline source (150) & 1000.000 & 1000.000 & 0.001 \\
phone (888) & 5.000 & 5.000 & 0.001 \\
phone (439) & 5.000 & 5.000 & 0.001 \\
phone (1331) & 5.000 & 5.000 & 0.001 \\
\hline
\end{tabular}

-

\begin{tabular}{||l|r|r|r||}
\hline \multicolumn{4}{|c||}{ position error estimate and standard deviation $(\mathrm{m})$} \\
instrument $(\operatorname{depth}(\mathrm{m}))$ & $x\left(\sigma_{x}\right)$ & $y\left(\sigma_{y}\right)$ & $z\left(\sigma_{z}\right)$ \\
\hline \hline source (150) & $-1791.16(305.22)$ & $413.7(975.52)$ & $0.00(0.00)$ \\
phone (888) & $-0.14(4.99)$ & $0.05(5.00)$ & $0.00(0.00)$ \\
phone (439) & $0.13(4.99)$ & $-0.05(5.00)$ & $0.00(0.00)$ \\
phone (1331) & $0.05(4.99)$ & $-0.02(5.00)$ & $0.00(0.00)$ \\
\hline
\end{tabular}

Table 4.1: The top panel shows the a priori standard deviations of the instrument locations. The bottom panel shows the position error estimates of the instruments used in the inversion with the associated standard deviations shown in parenthesis alongside.

by assigning the source the largest a priori standard deviation. The correction is significant in the $\mathrm{x}$-coordinate as it is much larger than the corresponding standard deviation. Therefore, the distance between the source and the receivers is greater than the initial guess.

For a significant sound-speed profile estimate, arrivals at all the hydrophones for multiple transmissions and/or more precise array navigation data are necessary. 


\section{Appendix A}

\section{The Gauss Markov theorem}

A proof of the Gauss Markov theorem [20] is outlined here for easy reference. Let the data, $d$, contain signal $G m$ and noise $n$,

$$
d=G m+n
$$

where the model parameters $m$ and the noise are zero mean stochastic processes. A linear estimate $\hat{\mathbf{m}}$ of $\boldsymbol{m}$ is sought. Explicitly, the estimate is to be linearly related to the data in the form, $\hat{\mathrm{m}}=\boldsymbol{L d}$.

The error in the estimate is $e=\hat{\mathrm{m}}-\boldsymbol{m}$. Minimizing the expected value of the squared error is equivalent to minimizing the diagonal elements of the error covariance matrix,

$$
C_{e e}=E\left[e e^{T}\right]=E\left[(L d-m)(L d-m)^{T}\right]
$$

where, $E[$.$] denotes taking the expectation. Expanding the previous expression yields,$

$$
C_{e e}=L E\left[d d^{T}\right] L^{T}-L E\left[d m^{T}\right]-E\left[m d^{T}\right] L^{T}+E\left[m m^{T}\right]
$$

Defining correlation matrices as $E\left[\boldsymbol{u} \boldsymbol{v}^{T}\right]=\boldsymbol{R}_{u v}$, the above expression becomes,

$$
C_{e e}=L R_{d d} L^{T}-L R_{m d}^{T}-R_{m d} L^{T}+R_{m m}
$$


Recalling a matrix identity [20] which states that for any $C=C^{T}$,

$$
\left(A-B C^{-1}\right) C\left(A-B C^{-1}\right)^{T}-B C^{-1} B^{T}=A C A^{T}-B A T-A B^{T}
$$

equation A.4 is equivalent to,

$$
C_{e e}=\left(L-R_{m d} R_{d d}^{-1}\right) R_{d d}\left(L-R_{m d} R_{d d}^{-1}\right)^{T}-R_{m d} R_{d d}^{-1} R_{m d}^{T}+R_{m m}
$$

Since the first two terms are quadratic forms and correlation matrices and their inverses are always positive semi-definite, the first two terms contribute non-negative diagonal elements to $C_{e e}$. Only the first term contains $L$, however. Choosing $\boldsymbol{L}=\boldsymbol{R}_{m d} \boldsymbol{R}_{d d}^{-1}$ makes the first term vanish, giving the linear minimum mean square estimate,

$$
\hat{\mathrm{m}}=R_{m d} R_{d d}^{-1} d
$$

with the error covariance,

$$
C_{e e}=R_{m m}-R_{m d} R_{d d}^{-1} R_{m d}^{T}
$$

One notes that if the model parameters and the data are uncorrelated, $\boldsymbol{R}_{\boldsymbol{m} d}=\mathbf{o}$,then $C_{e e}=R_{m m}$, which is consistent with the expectation that if the data contains no information about the model, then the error of the estimate is the same as the $a$ priori error.

Simplified expressions are obtained if the noise and model parameters are assumed uncorrelated ( $\boldsymbol{R}_{m n}=\mathbf{o}=\boldsymbol{R}_{n m}$ ). Then,

$$
R_{d d}=E\left[(G m+n)(G m+n)^{T}\right]=G R_{m m} G^{T}+R_{n n}
$$

and

$$
R_{m d}=E\left[m(G m+n)^{T}\right]=R_{m m} G^{T}
$$

Substituting equations A.9 and A.10 into A.7 and A.8, the simplified expressions for 
the estimate and error covariance are then,

$$
\hat{\mathrm{m}}=R_{m m} G^{T}\left(G R_{m m} G^{T}+R_{n n}\right)^{-1} d
$$

and

$$
C_{e e}=R_{m m}-R_{m m} G^{T}\left(G R_{m m} G^{T}+R_{n n}\right)^{-1} G R_{m m}^{T}
$$

The error covariance only involves the model kernel $G$ and the correlation matrices of the models and the noise.

The residual $\hat{\mathbf{n}}=\boldsymbol{d}-\boldsymbol{G} \hat{\mathrm{m}}$, after substituting in equation A.11, is

$$
\hat{\mathrm{n}}=d-G R_{m m} G^{T}\left(G R_{m m} G^{T}+R_{n n}\right)^{-1} d
$$

and after post multiplying the first term by the identity matrix and factoring out the $d$ vector,

$\hat{\mathbf{n}}=\left(\left(G R_{m m} G^{T}+R_{n n}\right)\left(G R_{m m} G^{T}+R_{n n}\right)^{-1}-G R_{m m} G^{T}\left(G R_{m m} G^{T}+R_{n n}\right)^{-1}\right) d$

Factoring out the inverted term this simplifies to,

$$
\hat{\mathrm{n}}=R_{n n}\left(G R_{m m} G^{T}+R_{n n}\right)^{-1} d
$$

This expression is the same expression obtained by writing down a Gauss Markov estimate of $\boldsymbol{n}$ in equation A.1, while viewing the $\boldsymbol{G} \boldsymbol{m}$ term as the "noise" and recalling that $\boldsymbol{n}$ and $\boldsymbol{m}$ are uncorrelated. The residual, therefore, is an estimate of the noise term. 


\section{Appendix B}

\section{3-D parameterization}

In this appendix we indicate how the 3-D parameterization is written in matrix form. The 3-D sound speed perturbation is parameterized as an expansion in vertical functions and horizontal functions, where the horizontal functions are now two dimensional fourier series,

$\delta c(x, y, z)=\sum_{i=1}^{L} a_{i 00} F_{i}(z)+\sum_{i=1}^{N} F_{i}(z)\left[\sum_{j=1}^{M} \sum_{l=1}^{L} a_{i j l} \cos \left(\frac{2 \pi j x}{R_{x}}+\frac{2 \pi l y}{R_{y}}\right)+b_{i j l} \sin \left(\frac{2 \pi j x}{R_{x}}+\frac{2 \pi l y}{R_{y}}\right)\right]$

where $R_{x}$ and $R_{y}$ are the periodicities of the horizontal sinusoidal functions in the $\mathrm{x}$ and y directions, respectively.

Substituting this expansion in 4.1 and moving the integral inside the summations yields,

$$
\begin{aligned}
\delta T_{k}= & -\sum_{i=1}^{L} a_{i 00} \int_{\Gamma(k)} \frac{F_{i}(z(s)) d s}{C_{o}^{2}(s)}-\sum_{i=1}^{N} \sum_{j=1}^{M} \sum_{l=1}^{L} a_{i j l} \int_{\Gamma(k)} \frac{F_{i}(z(s)) \cos \left(\frac{2 \pi j x(s)}{R_{x}}+\frac{2 \pi l y(s)}{R_{y}}\right) d s}{C_{o}^{2}(s)} \\
& -\sum_{i=1}^{N} \sum_{j=1}^{M} \sum_{l=1}^{L} b_{i j l} \int_{\Gamma(k)} \frac{F_{i}(z(s)) \sin \left(\frac{2 \pi j x(s)}{R_{x}}+\frac{2 \pi l y(s)}{R_{y}}\right) d s}{C_{o}^{2}(s)}+n_{k}
\end{aligned}
$$

To rewrite this expression in matrix form, the summations are broken up into terms indexed by the combinations of the $j$ and $l$ indices. Lets define,

$$
\psi_{j l}^{c}(k, i)=-\int_{\Gamma(k)} \frac{F_{i}(z(s)) \cos \left(\frac{2 \pi j x(s)}{R_{x}}+\frac{2 \pi l y(s)}{R_{y}}\right) d s}{C_{o}^{2}(s)}
$$




$$
\psi_{j l}^{s}(k, i)=-\int_{\Gamma(k)} \frac{F_{i}(z(s)) \sin \left(\frac{2 \pi j x(s)}{R_{x}}+\frac{2 \pi l y(s)}{R_{y}}\right) d s}{C_{o}^{2}(s)}
$$

and

$$
\boldsymbol{a}_{j l}=\left[\begin{array}{c}
a_{1 j l} \\
\vdots \\
a_{N j l}
\end{array}\right], \quad \boldsymbol{b}_{j l}=\left[\begin{array}{c}
b_{1 j l} \\
\vdots \\
b_{N j l}
\end{array}\right], \quad G_{j l}^{c}=\left[\psi_{j l}^{c}(k, i)\right], \quad G_{j l}^{s}=\left[\psi_{j l}^{s}(k, i)\right]
$$

The subscripting can be simplified. Make a one-to-one mapping to a new index $m$, where $m=(j-1) L+l$ for $1 \leq j \leq M, 1 \leq l \leq L$ and $m=0$ for $j=0=l$. Using the new subscript $m$, we can write,

$$
\begin{aligned}
& \psi_{m}^{c}(k, i)=-\int_{\Gamma(k)} \frac{F_{i}(z(s)) \cos \left(\frac{2 \pi j x(s)}{R_{x}}+\frac{2 \pi l y(s)}{R_{y}}\right) d s}{C_{o}^{2}(s)} \\
& \psi_{m}^{s}(k, i)=-\int_{\Gamma(k)} \frac{F_{i}(z(s)) \sin \left(\frac{2 \pi j x(s)}{R_{x}}+\frac{2 \pi l y(s)}{R_{y}}\right) d s}{C_{o}^{2}(s)}
\end{aligned}
$$

and

$$
\boldsymbol{a}_{m}=\left[\begin{array}{c}
a_{1 m} \\
\vdots \\
a_{N m}
\end{array}\right], \quad \boldsymbol{b}_{m}=\left[\begin{array}{c}
b_{1 m} \\
\vdots \\
b_{N m}
\end{array}\right], \quad G_{m}^{c}=\left[\psi_{m}^{c}(k, i)\right], \quad G_{m}^{s}=\left[\psi_{m}^{s}(k, i)\right]
$$

Appending these matrices appropriately,

$$
d=\left[\begin{array}{lllllll}
G_{\circ}^{c} & G_{1}^{c} & \ldots & G_{M L}^{c} & G_{1}^{s} & \ldots & G_{M L}^{s}
\end{array}\right]\left[\begin{array}{c}
a_{\circ} \\
a_{1} \\
\vdots \\
a_{M L} \\
b_{1} \\
\vdots \\
b_{M L}
\end{array}\right]+n
$$


Again, as in chapter 4 , the forward problem becomes,

$$
d=G_{a} m_{a}+n
$$

To visualize the construction of the model parameter vector, $\boldsymbol{m}_{a}$, let the parameters $a_{i j l}$ and $b_{i j l}$ form two 3-D lattices. First, from the $a_{i j l}$ lattice, the $a_{i 00}$ column is extracted. Then the first column of the $l=1$ plane in $a_{i j l}$ is stacked below that, followed by the second column and so on. Next the $l=2$ plane is stacked below the $l=1$ plane column by column and so on. Once the $a_{i j l}$ lattice is exhausted the columns of the $b_{i j l}$ lattice are extracted similarly and stacked below those of the $a_{i j l}$ parameters. 


\section{Bibliography}

[1] Spiesberger, J. L., and Metzger, K., Basin-Scale Tomography: A New Tool for Studying Weather and Climate, J. Geophys. Res. 96, 4869-4889 (1991).

[2] Munk, W., and C. Wunsch, Ocean Acoustic Tomography: A scheme for large scale monitoring, Deep-Sea Res., 26, 123-161, 1979.

[3] Robert Curtis Sparrock, "Stability of time fronts in a large vertical array at long range in the ocean," M.S. thesis, University of California, San Diego, Ca, 1990.

[4] B. J. Sotrin and J. A. Hildebrand, "Large aperture digital acoustic array," IEEE J. Oceanic Eng., 13, 271-282, 1988.

[5] B. J. Sotrin and J. A. Hildebrand, "Acoustic navigation of a large-aperture array," J. Acoust. Soc. Am., 87, 154-167, 1990.

[6] Spiesberger, J. L., and Metzger, K., "A New Algorithm for Sound Speed in Seawater," J. Acoust. Soc. Am., 89(6), June 1991.

[7] Metzger, K. Jr., "Signal Processing Equipment and Techniques for Use in Measuring Acoustic Multipath Structures," Ph.D. Thesis, Cooley Electronics Laboratory, University of Michigan, Ann Arbor, MI, (1983).

[8] Metzger, K. Jr., computer code MST, University of Michigan, Ann Arbor, MI, 1989.

[9] B. Elspas, "The theory of autonomous linear sequential networks," IRE Transactions on Circuit Theory, Vol. CT-6, No. 1, March 1959.

[10] S. W. Golomb, Shift Register Sequences, Holden-Day, San Francisco, California, 1967

[11] Martin Cohn and Abraham Lempel, "On Fast M-sequence Transforms," IEEE Transactions on Information Theory, January 1977.

[12] Jeffrey Borish and James B. Angell, "An Efficient Algorithm for Measuring the Impulse Response using Pseudorandom noise," J. Audio Eng. Soc., Vol. 31, No. $7,1983$.

[13] Metzger, K. Jr., and Bouwens, R. J., "An Ordered Table of Primitive Polynomials over GF(2) of degrees 2 through 19 for use with Linear Maximal Sequence Generators," Cooley Electronics Laboratory Technical Memorandum No. 107, The University of Michigan, July 1972. 
[14] Hamilton K. G., Siegmann W. L., and Jacobson M. J., "Simplified calculation of ray-phase perturbations due to ocean- environmental variations, J. Acoust. Soc. Am.,vol. 67, pp. 1193-1206, Apr. 1980

[15] Spiesberger, J. L., "Ocean acoustic tomography: travel time biases," J. Acoust. Soc. Am., 77, 83-100, 1985.

[16] Spiesberger, J. L., and P. F. Worcester, "Perturbations in travel time and ray geometry due to mesoscale disturbances: a comparison of exact and approximate calculations," J. Acoust. Soc. Am., 74,219-225, 1983.

[17] Cook, Charles E., and Bernfeld, Marvin, Rader signals, Academic Press, New York, NY, 1967.

[18] Brekhovskikh, L., and Lysanov, Y., Fundamentals of Ocean Acoustics, SpringerVerlag, Berlin Heidelberg, 1982.

[19] Aki, K. and P. G. Richards, Quantitative Seismology: Theory and Methods, Vol. II, W. H. Freeman, San Francisco, Calif., 1980.

[20] Liebelt, P. B., An Introduction to Optimal Estimation, Addison-Wesley, 1967.

[21] Bruce D. Cornuelle, "Simulations of Acoustic Tomography Array performance with Untracked or Drifting Sources and Receivers," J. Geophysical Res., Vol. 90, 9079-9088, September 20, 1985.

[22] Levitus, S., "Climatological atlas of the world ocean," NOAA prof. Paper No. 13, U.S. Government Printing Office, Washington DC, 1982.

[23] Del Grosso, V. A., "New equation for the speed of sound in natural waters (with comparisons to other equations)," J. Acous. Soc. Am., 56, 1084-1091, 1974.

[24] C. Chen, F. J. Millero, "Speed of sound in seawater at high pressures," J. Acous. Soc. Am., 62, 1129-1135, 1977

[25] L. Boden, J. B. Bowlin, J. L. Spiesberger, "Time domain analysis of normal mode, parabolic, and ray solutions of the wave equation," J. Acous. Soc. Am., 90, 954-958, 1991.

[26] Bruce D. Cornuelle, private communication. 